\title{
Cholinesterase level is a predictor of systemic inflammatory response syndrome and complications after cardiopulmonary bypass
}

\author{
Yi Yang, Xiaolei Yang, Jin Yang \\ Department of Intensive Care Unit, Chongqing General Hospital, University of Chinese Academy of Sciences, Chongqing, China \\ Contributions: (I) Conception and design: Y Yang; (II) Administrative support: J Yang; (III) Provision of study materials or patients: X Yang; (IV) \\ Collection and assembly of data: X Yang; (V) Data analysis and interpretation: Y Yang; (VI) Manuscript writing: All authors; (VII) Final approval of \\ manuscript: All authors. \\ Correspondence to: Jin Yang. No. 312, Zhongshan Road, Yuzhong District, Chongqing 400013, China. Email: cb0750@163.com.
}

Background: To study the changes of serum cholinesterase (S-ChE) levels in patients with systemic inflammatory response syndrome (SIRS) after cardiopulmonary bypass (CPB) and its predictive value for multiple organ dysfunction syndrome (MODS).

Methods: Forty-two patients with SIRS after CPB were selected as the SIRS group. Another 42 patients who did not develop SIRS after CPB were selected as the control group. The S-ChE levels of patients were compared between the two groups at the time of intensive care unit (ICU) admission and 48 hours after admission to the ICU. The correlation between S-ChE levels and Acute Physiology and Chronic Health EvaluationII (APACHEII) score, tumor necrosis factor- $\alpha$ (TNF- $\alpha$ ), and interleukin-6 (IL-6) levels was compared between the two groups at the time of ICU admission and 48 hours after ICU admission, and receiver operator characteristic (ROC) curve analysis was used to analyze the accuracy of S-ChE level in diagnosing MODS in patients with SIRS after CPB.

Results: There was no significant difference in the S-ChE levels among the non-SIRS patients between the time of admission to the ICU and 48 hours later. However, the S-ChE levels in SIRS patients exhibited a decreasing trend from 48 hours after ICU admission compared with those at the time of ICU admission. Meanwhile, the S-ChE levels were obviously lower in SIRS patients than in non-SIRS patients at those two time points. Furthermore, we found that S-ChE levels were negatively correlated with APACHEII scores as well as the levels of TNF- $\alpha$ and IL-6 at ICU admission and 48 hours after ICU admission in SIRS patients. Meanwhile, S-ChE levels were associated with the occurrence of MODS of SIRS patients. Subsequent ROC curve analysis revealed that early S-ChE levels could predict the occurrence of SIRS complicated by MODS after CBP.

Conclusions: S-ChE was significantly decreased in patients with SIRS after CPB, and the decrease of S-ChE in the early stage was closely related to the severity of SIRS after CBP and the increase of inflammatory cytokines. Meanwhile, there was a close relationship between S-ChE activity and MODS in the early stage.

Koywords: Cholinesterase; cardiopulmonary bypass (CPB); systemic inflammatory response syndrome (SIRS)

Submitted Sep 11, 2021. Accepted for publication Oct 29, 2021.

doi: 10.21037/apm-21-2889

View this article at: https://dx.doi.org/10.21037/apm-21-2889 


\section{Introduction}

The development of cardiac surgery depends on the progress of cardiopulmonary bypass (CPB). However, $\mathrm{CPB}$ can disturb the homeostasis of the human body, cause nervous and endocrine system disorders, and lead to systemic inflammatory response syndrome (SIRS), which is the main factor in the development of postoperative complications $(1,2)$. SIRS $=$ the systemic inflammatory response to a variety of severe clinical insults. The response is manifested by two or more of the following conditions: (I) temperature 38 or $<36{ }^{\circ} \mathrm{C}$; (II) heart rate $>90$ beats per minute; (III) respiratory rate $>20$ breaths per minute or $\mathrm{PaCO}_{2}<32 \mathrm{mmHg}$; and (IV) white blood cell count $>12,000$ Icumm, $<4,000 /$ cumm, or $>10 \%$ immature (band) forms (3). The development of SIRS can lead to multiple organ dysfunction syndrome (MODS), which seriously affects the prognosis of patients and even leads to death. $\mathrm{CPB}$ can induce SIRS through a variety of mechanisms, including blood contact with artificial materials to activate complements and cytokines, ischemia-reperfusion injury of multiple organs during $\mathrm{CPB}$, endotoxemia caused by ectopic intestinal bacteria and viruses, trauma and body temperature changes caused by surgery and the release of nitric oxide (NO), etc. (4). Cholinesterase (ChE) in the human body is mainly divided into two types: acetylcholinesterase (AChE) and butyrylcholinesterase (BChE). AChE is a key enzyme in biological nerve conduction, which can degrade acetylcholine (Ach), terminate the excitatory effect of neurotransmitters on the postsynaptic membrane in time, and ensure the normal transmission of neural signals. The cholinergic system has been shown to exert antiinflammatory effects by regulating the immune system through non-neuronal Ach $(5,6)$, while the decrease of BChE activity can be used as an early indicator of acute systemic inflammation (7). There is a report on a marked reduction in previously normal $\mathrm{ChE}$ activity after CPB (8). However, the changes of serum cholinesterase (S-ChE) in SIRS after CPB are rarely reported. This study aims to investigate the changes of early $\mathrm{S}-\mathrm{ChE}$ in patients with SIRS after $\mathrm{CPB}$, and its correlation with disease severity degree and inflammatory cytokine levels. Furthermore, we aim to evaluate its predictive value for the development of MODS. We present the following article in accordance with the STARD reporting checklist (available at https:// dx.doi.org/10.21037/apm-21-2889).

\section{Methods}

\section{General information}

In this study, we selected a total of 84 patients with rheumatic heart disease who received heart valve replacement or valvuloplasty under CPB in Chongqing General Hospital from May 2019 to May 2021. The patients were divided into two groups according to the diagnostic criteria of SIRS (9) and whether the patients developed SIRS after surgery: a SIRS group ( $n=42)$ and a control group $(n=42)$. The SIRS group was further divided into a MODS group $(\mathrm{n}=19)$ and a NO-MODS group $(n=23)$ according to the diagnostic criteria of MODS (10).

Inclusion criteria: (I) patients with rheumatic heart disease who were scheduled to undergo heart valve replacement under $\mathrm{CPB}$; and (II) patients aged 18 to 75 years old.

Exclusion criteria: (I) patients with a preoperative and postoperative 24-hour left ventricular ejection fraction (LVEF) $<35 \%$; (II) patients combined with diabetes, organ dysfunction, tumor, or immune system disease; (III) patients with acute lung injury before emergency surgery; and (IV) patients with acute infection, hepatitis, liver cirrhosis, and organophosphorus or carbamates pesticide poisoning.

The age, gender, past history, medication, vital signs, and other general conditions of all cases were recorded. All procedures performed in this study involving human participants were in accordance with the Declaration of Helsinki (as revised in 2013). All patients had signed an informed consent form, and this study was approved by the Committee of Chongqing General Hospital (No. S2019047-01).

\section{S-CbE detection}

Serum samples were collected after CPB, upon admission to intensive care unit (ICU), and after 48 hours. S-ChE was detected using an automatic biochemical analyzer (BS280, Mindray, China) using the rate method. The normal reference value is $4,500-13,000 \mathrm{U} / \mathrm{L}$.

\section{Enzyme-linked immunosorbent assay (ELISA)}

The serum of patients was collected at the time of ICU admission and 48 hours later, and the levels of interleukin 
Table 1 Comparison of general data between the two groups

\begin{tabular}{lcccc}
\hline Variable & Control group $(\mathrm{n}=42)$ & SIRS group $(\mathrm{n}=42)$ & $\chi^{2} / t$ & $\mathrm{P}$ \\
\hline Gender (females, \%) & $28(66.7 \%)$ & $25(59.2 \%)$ & 0.104 & 0.951 \\
Age (years) & $46.9 \pm 9.2$ & $48.3 \pm 9.5$ & 0.983 & 0.342 \\
BMI (kg/m $\left.{ }^{2}\right)$ & $23.56 \pm 3.45$ & $21.26 \pm 2.15$ & 1.243 & 0.219 \\
LVEF (\%) & $54.23 \pm 6.45$ & $52.56 \pm 7.58$ & 1.429 & 0.307 \\
CPB time (min) & $141.45 \pm 28.53$ & $146.27 \pm 26.48$ & 1.385 & 0.237 \\
ACCT (min) & $82.15 \pm 38.22$ & $86.27 \pm 29.58$ & 1.262 & 0.214 \\
ACT (min) & $31.21 \pm 13.48$ & $29.65 \pm 9.73$ & 1.137 & 0.185 \\
\hline
\end{tabular}

BMI, body mass index; LVEF, left ventricular ejection fraction; CPB, cardiopulmonary bypass; ACCT, aortic cross-clamp time; ACT, auxiliary cycle time.

(IL)-6 and tumor necrosis factor (TNF)- $\alpha$ in serum were measured using ELISA kits (Cayman Bio, USA).

\section{Acute physiology and chronic bealth evaluation score}

The patients underwent routine postoperative examination, and clinical parameters such as C-reactive protein (CRP), platelet count, blood gas analysis, liver and kidney function parameters, oxygenation index $\left(\mathrm{PaO}_{2} / \mathrm{FiO}_{2}\right)$, heart rate (HR), central venous pressure (CVP), blood pressure (BP), and so on were recorded. The Acute Physiology and Chronic Health EvaluationII (APACHEII)score was evaluated according to the APACHEII scoring rules (8).

\section{Statistical methods}

SPSS 21.0 software (SPSS, Inc., Chicago, IL, USA) was used for statistical analysis, and measurement data were expressed as mean \pm standard deviation (SD). Comparisons between two groups were analyzed by the $t$-test. Enumeration data were analyzed by the chi-square $\left(\chi^{2}\right)$ test and correlation analysis was performed using Pearson analysis. The receiver operating characteristic (ROC) curve was used to analyze the accuracy of S-ChE levels after CPB at the time of ICU admission and 48 hours later to predict SIRS patients complicated with MODS after $\mathrm{CPB}$. $\mathrm{P}<0.05$ was considered statistically significant.

\section{Results}

\section{General information of patients}

The ages of the subjects ranged from 44 to 78 (47.78
12.24) years. Our study cohort included 52 males and 32 females. Among them, 31 patients underwent mitral valve replacement, 28 patients underwent aortic valve replacement, and 25 patients underwent both mitral valve replacement and aortic valve replacement. There were no statistically significant differences in age, gender, body mass index (BMI), CPB time, aortic cross-clamp time (ACCT), and auxiliary cycle time (ACT) between the two groups of patients (Table 1).

\section{Levels of $S-C b E$ were decreased after $C P B$ in patients with SIRS}

The S-ChE levels of the two groups were detected upon admission to the ICU and at 48 hours after admission to ICU. The results showed that there was no significant change in the S-ChE level between these two time points. However, the level of S-ChE in SIRS group decreased significantly upon admission to the ICU and 48 hours later, and the level of S-ChE at 48 hours later was markedly lower than that at the time of admission to ICU $(\mathrm{P}<0.01$, Figure 1).

\section{There was a negative correlation between the S-ChE level and the APACHEEII score in patients with SIRS}

The APACHEII score is a widely used scoring system in ICUs around the world, which can indicate abnormal changes in the detection and treatment of acute physiology. In order to study the clinical significance of changes in S-ChE levels, we used Pearson correlation analysis to evaluate the correlation between S-ChE levels and APACHEII scores in patients with SIRS upon admission to the ICU. The results showed that there was a negative 


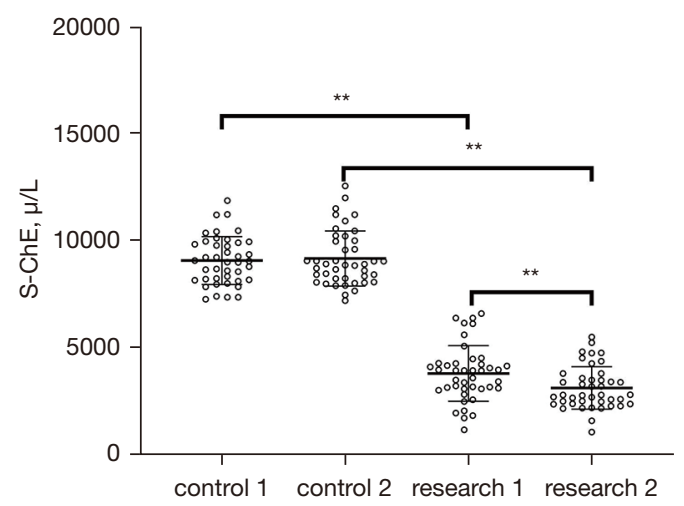

Figure 1 Comparison of S-ChE levels between the two groups at different time points Control 1: control group at the time of admission to the ICU. Control 2: control group at 48 hours after admission to the ICU. Research 1: SIRS group at the time of admission to the ICU. Research 2: SIRS group on 48 hours after admission to the ICU. **, $\mathrm{P}<0.01$. S-ChE, serum cholinesterase; ICU, intensive care unit; SIRS, systemic inflammatory response syndrome.

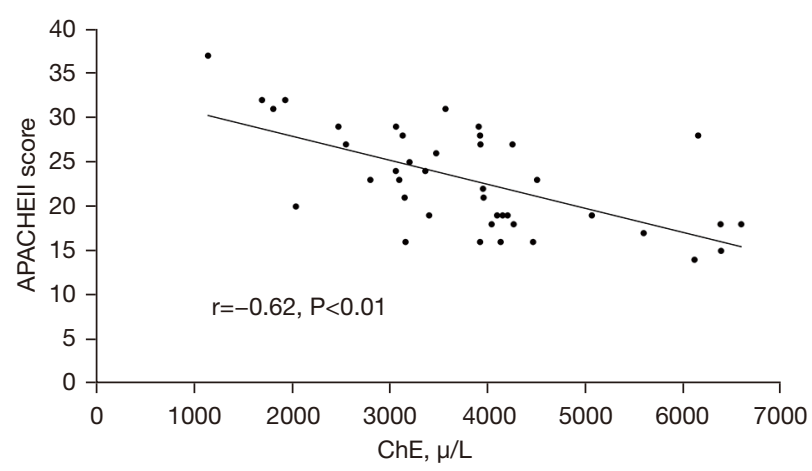

Figure 2 Correlation between S-ChE and the APACHEII score in patients with SIRS at the time of ICU admission. S-ChE, serum cholinesterase; SIRS, systemic inflammatory response syndrome; $\mathrm{ICU}$, intensive care unit.

correlation between the S-ChE level and the APACHEII score $(\mathrm{r}=0.62, \mathrm{P}<0.01$, Figure 2$)$.

\section{Cholinesterase was negatively correlated with IL-6 and TNF- $\alpha$ in patients with SIRS at the time of admission to ICU}

Since TNF- $\alpha$ and IL-6 are important cytokines involved in the occurrence of SIRS after CPB, Pearson correlation analysis was used to analyze the correlation between S-ChE,
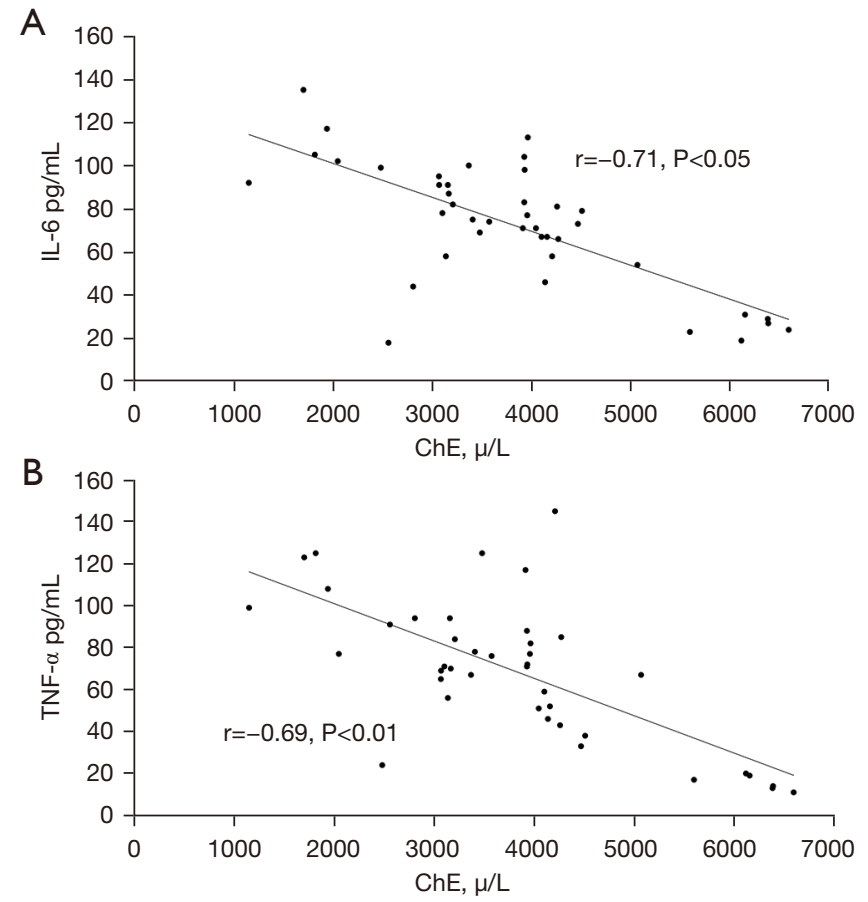

Figure 3 The correlation between S-ChE, IL-6, and TNF- $\alpha$ upon admission of SIRS patients to the ICU. (A) The correlation between S-ChE and IL-6 upon admission of SIRS patients to the ICU. (B) The correlation between S-ChE and TNF- $\alpha$ upon admission of SIRS patients to the ICU. S-ChE, serum cholinesterase; IL, interleukin; TNF, tumor necrosis factor; SIRS, systemic inflammatory response syndrome; ICU, intensive care unit.

IL-6, and TNF- $\alpha$ upon admission of SIRS patients to the ICU. The results suggested that there was a negative correlation between S-ChE and IL-6 (0.71) and TNF- $\alpha$ (0.69) in serum (Figure 3).

\section{$S-C b E$ levels in SIRS patients were related to the occurrence of MODS}

In order to further investigate the correlation between the occurrence of MODS in SIRS patients and SIRS-related factors, we performed routine examination upon admission of patients to the ICU, and measured the levels of S-ChE in MODS and NO-MODS groups at the same time. The results indicated that the level of S-ChE in MODS group was significantly lower than that in NO-MODS group, but there was no significant difference in oxygenation index $(\mathrm{PaO} 2 / \mathrm{FiO} 2)$, pressure-adjusted heart rate $(\mathrm{PAR})=$ heart rate $(\mathrm{HR}) \times$ central venous pressure $(\mathrm{CVP}) /$ mean artery pressure $(\mathrm{MAP})$, creatinine $(\mathrm{Cr})(\mu \mathrm{mol} / \mathrm{L})$, platelet count, 
Table 2 Comparison of $\mathrm{PaO}_{2} / \mathrm{FiO}_{2}$, PAR, Cr, LVEF, PLT, PH, S-ChE between MODS and NO-MODS group

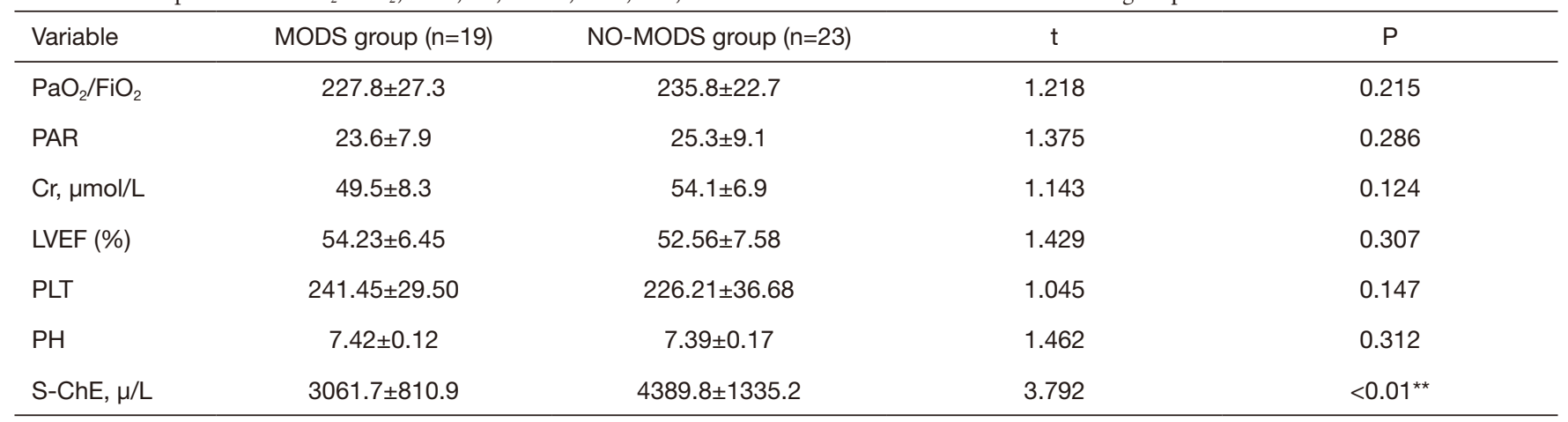

$\mathrm{PAR}=\mathrm{HR} \times \mathrm{CVP} / \mathrm{MAP} ;{ }^{*}, \mathrm{P}<0.01 . \mathrm{PaO}_{2} / \mathrm{FiO}_{2}$, oxygenation index; PAR, pressure-adjusted heart rate; LVEF, left ventricular ejection fraction; $\mathrm{PLT}$, platelet; $\mathrm{PH}$, potential of hydrogen; $\mathrm{S}-\mathrm{ChE}$, serum cholinesterase; MODS, multiple organ dysfunction syndrome.

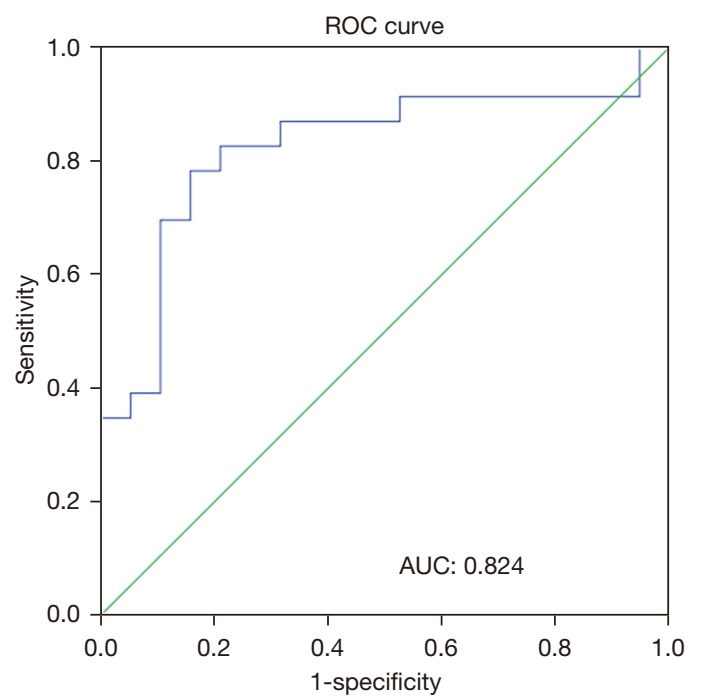

Figure 4 ROC analysis for predicting MODS upon ICU admission in SIRS patients. ROC, receiver operating characteristic; MODS, multiple organ dysfunction syndrome; ICU, intensive care unit; SIRS, systemic inflammatory response syndrome.

and potential of hydrogen $(\mathrm{PH})$ value (Table 2).

\section{Change of S-CbE level in the early stage could predict the occurrence of MODS in patients with SIRS}

ROC curve analysis of S-CHE level upon admission of patients to the ICU was used to evaluate the predictive value of MODS in patients with SIRS. The results showed that the receiver operator characteristic (AUC) of early S-ChE was 0.824 [95\% confidence interval (CI): 0.6900.958; $\mathrm{P}=0.000]$. The best predicted value was 3,098 uamp $\mathrm{L}$, with a sensitivity of $78 \%$ and a specificity of $84 \%$ (Figure 4). These results suggested that the level of early S-CHE could be used as a predictor of MODS in patients with SIRS.

\section{Discussion}

Traditionally, S-ChE has been mainly used to diagnose organophosphorus pesticide poisoning and assess the status of liver function. However, in recent years, an increasing number of researchers have found that $\mathrm{S}-\mathrm{ChE}$ may be important in some diseases, especially SIRS. As early as 1979, Oestern et al. (11) studied 50 patients with severe multiple trauma and found that S-ChE activity was obviously lower in those who died compared to the survivors. al-Kassab et al. (12) monitored S-ChE activity in 26 patients with septic shock in ICU for 7 days and found that S-ChE activity was significantly declined compared with the normal control group, and the decrease was persistent. Kanai et al. (13) also found that S-ChE activity was notably reduced in patients with bacteremia compared to patient groups with various bacterial infections who did not develop bacteremia. They concluded that S-ChE activity was reduced more rapidly when bacteremia occurred in patients with bacterial infections, and thus, sepsis could be detected early. Zivkovic et al. (7) suggested that a prominent decrease in BChE activity could be detected in patients after minor trauma at the time of ICU admission, which can rapidly and sensitively detect emerging systemic inflammation by detecting the activity of BChE. In 2000, Borovikova et al. (5) first proposed the concept of the cholinergic anti-inflammatory pathway (CAP), and found a parasympathetic anti-inflammatory pathway through which the brain regulates the systemic inflammatory response 
(caused by endotoxin).

Inflammatory mediators, such as IL- $1 \beta$, IL-6, IL18 , and TNF, inhibit their pro-inflammatory activity in a feedback manner by stimulating the vagus nerve to activate the cholinergic system. The changes in ChE activity and the pathophysiological mechanisms of systemic inflammation may be related to the anti-inflammatory activity of the non-neuronal cholinergic system (14-16). So, activating cholinergic anti-inflammatory pathway may be a treatment of SIRS after CPB. It has been shown (17) that the production of $\mathrm{BChE}$ and albumin in the liver is coupled, and these biochemical variables may be considered as negative inflammatory reactants whose serum levels are inversely correlated with the increase in the degree of clinical inflammation. Cholinergic parameters are associated with a variety of inflammatory markers, including fibrinogen, IL-6, and CRP (18). The measurement of the S-ChE activity can be used to quickly assess the nonneuronal cholinergic activity upon an inflammatory response (19). de Oliveira et al. (20) showed that there is a cell-specific mechanism for regulating the expression of AChE in inflammation. Meanwhile, TNF- $\alpha$ and IL-6 are important cytokines involved in the development of SIRS after CPB (4). In this study, we found for the first time that the level of S-ChE of patients with SIRS was significantly lower than that in patients without SIRS after CPB. The level of S-ChE in the SIRS group tended to be prominently decreased in the early stage (at the time of ICU admission), while there was no notable change in patients without SIRS within 48 hours after admission to the ICU. A significant negative correlation was also identified between S-ChE levels and the cytokines TNF- $\alpha$ and IL-6.

The APACHEII score is a universally applied method to evaluate diseases in ICU. Our study showed that there was a strong negative correlation between $\mathrm{S}-\mathrm{ChE}$ damage and the APACHEII score, which was similar to the findings of Zhang et al. (21). If SIRS is not effectively controlled, its further development can lead to MODS and significantly multiply the mortality (22). Thus, the prediction of MODS also plays an important role in controlling SIRS mortality. Some studies have reported that $\mathrm{S}-\mathrm{ChE}$ is associated with short-term mortality in septic patients who develop systemic inflammation (23). In this study, we also found that the decrease of S-ChE in the early stage could predict the risk of concurrent MODS in patients in the short term postoperatively.

However, in this study, the mechanism was not explored in depth, and only preliminary speculation was made from previous studies. Moreover, the sample size in this study was small, no data on long-term prognosis were collected, and the relationship between S-ChE activity changes and prognosis was not investigated in depth.

\section{Conclusions}

In summary, we found that S-ChE levels were significantly lower in patients with SIRS after CBP, and that reduced $\mathrm{S}$-ChE levels in the early stage were closely related to the severity of SIRS after CBP and increased inflammatory cytokines. We also confirmed that a lower early S-ChE increased the likelihood that SIRS patients were complicated by MODS, which provides a new research direction and theoretical basis for the treatment and improvement of prognosis in SIRS patients.

\section{Acknowledgments}

Funding: None.

\section{Footnote}

Reporting Checklist: The authors have completed the STARD reporting checklist. Available at https://dx.doi. org/10.21037/apm-21-2889

Data Sharing Statement: Available at https://dx.doi. org/10.21037/apm-21-2889

Conflicts of Interest: All authors have completed the ICMJE uniform disclosure form (available at https://dx.doi. org/10.21037/apm-21-2889). The authors have no conflicts of interest to declare.

Ethical Statement: The authors are accountable for all aspects of the work in ensuring that questions related to the accuracy or integrity of any part of the work are appropriately investigated and resolved. All procedures performed in this study involving human participants were in accordance with the Declaration of Helsinki (as revised in 2013). All patients had signed an informed consent form, and this study was approved by the Committee of Chongqing General Hospital (No. S2019-047-01).

Open Access Statement: This is an Open Access article distributed in accordance with the Creative Commons Attribution-NonCommercial-NoDerivs 4.0 International 
License (CC BY-NC-ND 4.0), which permits the noncommercial replication and distribution of the article with the strict proviso that no changes or edits are made and the original work is properly cited (including links to both the formal publication through the relevant DOI and the license). See: https://creativecommons.org/licenses/by-nc-nd/4.0/.

\section{References}

1. Paparella D, Yau TM, Young E. Cardiopulmonary bypass induced inflammation: pathophysiology and treatment. An update. Eur J Cardiothorac Surg 2002;21:232-44.

2. Holmes JH 4th, Connolly NC, Paull DL, et al. Magnitude of the inflammatory response to cardiopulmonary bypass and its relation to adverse clinical outcomes. Inflamm Res 2002;51:579-86.

3. Bone RC, Balk RA, Cerra FB, et al. Definitions for sepsis and organ failure and guidelines for the use of innovative therapies in sepsis. The ACCP/SCCM Consensus Conference Committee. American College of Chest Physicians/Society of Critical Care Medicine. Chest 1992;101:1644-55.

4. Larmann J, Theilmeier G. Inflammatory response to cardiac surgery: cardiopulmonary bypass versus noncardiopulmonary bypass surgery. Best Pract Res Clin Anaesthesiol 2004;18:425-38.

5. Borovikova $L V$, Ivanova $S$, Zhang $M$, et al. Vagus nerve stimulation attenuates the systemic inflammatory response to endotoxin. Nature 2000;405:458-62.

6. Tracey KJ. The inflammatory reflex. Nature 2002;420:853-9.

7. Zivkovic AR, Bender J, Brenner T, et al. Reduced butyrylcholinesterase activity is an early indicator of trauma-induced acute systemic inflammatory response. J Inflamm Res 2016;9:221-30.

8. Diefenbach C, Abel M, Rump AF, et al. Changes in plasma cholinesterase activity and mivacurium neuromuscular block in response to normothermic cardiopulmonary bypass. Anesth Analg 1995;80:1088-91.

9. Goldstein B, Giroir B, Randolph A, et al. International pediatric sepsis consensus conference: definitions for sepsis and organ dysfunction in pediatrics. Pediatr Crit Care Med 2005;6:2-8.

10. Zhang N, Shi F, Liang H, et al. The feasibility of using Hcy, CRP, and Cys-C to analyze AMI patients' disease conditions and prognoses. Am J Transl Res 2021;13:2724-30.

11. Oestern HJ, Trentz O, Hempelmann G, et al. Cardiorespiratory and metabolic patterns in multiple trauma patients. Resuscitation 1979;7:169-83.
12. al-Kassab AS, Vijayakumar E. Profile of serum cholinesterase in systemic sepsis syndrome (septic shock) in intensive care unit patients. Eur J Clin Chem Clin Biochem 1995;33:11-4.

13. Kanai S, Honda T, Uehara T, et al. Liver function tests in patients with bacteremia. J Clin Lab Anal 2008;22:66-9.

14. Rosas-Ballina M, Tracey KJ. Cholinergic control of inflammation. J Intern Med 2009;265:663-79.

15. Andersson U, Tracey KJ. Neural reflexes in inflammation and immunity. J Exp Med 2012;209:1057-68.

16. Andersson U, Tracey KJ. Reflex principles of immunological homeostasis. Annu Rev Immunol 2012;30:313-35.

17. Lampón N, Hermida-Cadahia EF, Riveiro A, et al. Association between butyrylcholinesterase activity and lowgrade systemic inflammation. Ann Hepatol 2012;11:356-63.

18. Ben Assayag E, Shenhar-Tsarfaty S, Ofek K, et al. Serum cholinesterase activities distinguish between stroke patients and controls and predict 12-month mortality. Mol Med 2010;16:278-86.

19. Zivkovic AR, Schmidt K, Stein T, et al. Bedsidemeasurement of serum cholinesterase activity predicts patient morbidity and length of the intensive care unit stay following major traumatic injury. Sci Rep 2019;9:10437.

20. de Oliveira P, Gomes AQ, Pacheco TR, et al. Cellspecific regulation of acetylcholinesterase expression under inflammatory conditions. Clin Hemorheol Microcirc 2012;51:129-37.

21. Zhang QH, Li AM, He SL, et al. Serum Total Cholinesterase Activity on Admission Is Associated with Disease Severity and Outcome in Patients with Traumatic Brain Injury. PLoS One 2015;10:e0129082.

22. Jouan J, Golmard L, Benhamouda N, et al. Gene polymorphisms and cytokine plasma levels as predictive factors of complications after cardiopulmonary bypass. J Thorac Cardiovasc Surg 2012;144:467-73, 473.e1-2.

23. Peng ZL, Huang LW, Yin J, et al. Association between early serum cholinesterase activity and 30-day mortality in sepsis-3 patients: A retrospective cohort study. PLoS One 2018;13:e203128.

(English Language Editor: A. Kassem)

Cite this article as: Yang Y, Yang X, Yang J. Cholinesterase level is a predictor of systemic inflammatory response syndrome and complications after cardiopulmonary bypass. Ann Palliat Med 2021;10(11):11714-11720. doi: 10.21037/apm-21-2889 